\title{
Risk Factors, Characteristics, and Outcome in Non-Ventilated Patients with Spontaneous Pneumothorax or Pneumomediastinum Associated with SARS-CoV-2 Infection
}

\author{
Adina Maria Marza (iD) ${ }^{1,2}$ \\ Alina Petrica (D) 1,3 \\ Diana Lungeanu (iD ${ }^{4}$ \\ Dumitru Sutoi ${ }^{1,5}$ \\ Alexandra Mocanu ${ }^{6}$ \\ loan Petrache ${ }^{1,7}$ \\ Ovidiu Alexandru Mederle 1,2 \\ 'Department of Surgery, "Victor Babes" \\ University of Medicine and Pharmacy, \\ Timisoara, 30004I, Romania; ${ }^{2}$ Emergency \\ Department, Emergency Clinical Municipal \\ Hospital, Timisoara, 300079, Romania; \\ ${ }^{3}$ Emergency Department, "Pius Brinzeu" \\ Emergency Clinical County Hospital, \\ Timisoara, 300736, Romania; ${ }^{4}$ Center for \\ Modeling Biological Systems and Data \\ Analysis, Department of Functional Sciences, \\ "Victor Babes" University of Medicine and \\ Pharmacy, Timisoara, 30004I, Romania; \\ ${ }^{5}$ Clinic of Anaesthesia and Intensive Care, \\ "Pius Brinzeu" Emergency Clinical County \\ Hospital, Timisoara, 300736, Romania; \\ ${ }^{6}$ Department of Infectious Diseases, "Victor \\ Babes" University of Medicine and Pharmacy, \\ Timisoara, 30004I, Romania; ${ }^{7}$ Clinic of \\ Thoracic Surgery, Emergency Clinical \\ Municipal Hospital, Timisoara, 300079, \\ Romania
}

Correspondence: Alina Petrica

Department of Surgery, "Victor Babes"

University of Medicine and Pharmacy, Piata

Eftimie Murgu 2, Timisoara, 30004I,

Romania

Tel +40744772427

Email alina.petrica@urgentatm.ro

Diana Lungeanu

Center for Modeling Biological Systems and

Data Analysis, Department of Functional

Sciences, "Victor Babes" University of

Medicine and Pharmacy, Piata Eftimie Murgu

2, Timisoara, 30004I, Romania

Email dlungeanu@umft.ro
Background and Objectives: Spontaneous pneumothorax (SP) and spontaneous pneumomediastinum (SPM) have frequently been cited as complications associated with coronavirus disease 2019 (COVID-19) pneumonia, with especially poor prognosis in mechanically ventilated patients. The current literature is controversial regarding the potential risk factors for developing SP or SPM (SP-SPM) in non-ventilated COVID-19 patients. Our research addressed a twofold objective: (a) to investigate the characteristics of patients with SP-SPM (both with and without COVID-19) and compare them to patients with sole COVID-19; (b) to quantify the risk of in-hospital mortality associated with SP-SPM and COVID-19.

Patients and Methods: A retrospective case-control study was conducted in the emergency departments (ED) of two tertiary hospitals in Timisoara, Romania, over one year (1st April 2020-31st March 2021; 64,845 records in total) and 70 cases of SP-SPM were identified (both SARS-CoV-2 positives and negatives). The control group comprised COVID-19 patients with no SP-SPM, included at a 2:1 ratio. Logistic regression was employed to quantify the in-hospital mortality risk associated with age, SP-SPM, and COVID-19.

Results: SP-SPM and COVID-19 were connected with prolonged hospitalization, a higher percentage of intensive care admission, and a higher mortality. SP-SPM increased the odds of death by almost four times in patients of the same age, gender, smoking status, and SARSCoV-2 infection: OR $=3.758,95 \%$ CI (1.443-9.792). Each additional year of age added $9.4 \%$ to the mortality risk: $\mathrm{OR}=1.094,95 \% \mathrm{CI}(1.054-1.135)$.

Conclusion: ED physicians should acknowledge these potential risks when attending COVID-19 patients with SP-SPM.

Keywords: case-control study, in-hospital mortality, chest pain, dyspnea, chest CT, COVID-19

\section{Introduction}

Spontaneous pneumothorax (SP) and spontaneous pneumomediastinum (SPM) are potential complications of some pulmonary disorders, such as chronic obstructive pulmonary disease (COPD), tuberculosis, lung cancer, and Pneumocystis jirovecii pneumonia. ${ }^{1}$ They are also frequently reported in mechanically ventilated patients with acute respiratory distress syndrome (ARDS) and severe acute respiratory syndrome-coronavirus 2 (SARS-CoV-2) infection, which was first reported in Wuhan, Hubei Province, China, in December $2019 .^{2-5}$ 
The incidence of SP or SPM (SP-SPM) in patients with COVID-19, the respiratory disease caused by SARS-CoV2 , is currently unknown. A large study by Miró et $\mathrm{al}^{6}$ reported a small number of patients with SP and COVID-19, with an incidence of less than 1\%. Other retrospective studies on patients with COVID-19 suggested that pneumothorax occurred in $1 \%$ of patients requiring hospital admission, $2 \%$ of those requiring intensive care unit (ICU) admission, and $1 \%$ of those dying of the infection. ${ }^{7}$ A systematic review recently published by Chong et $\mathrm{al}^{8}$ highlighted a low incidence of pneumothorax in hospitalized COVID-19 patients (0.3\%), but that increased to $12.8-23.8 \%$ for those who were mechanically ventilated.

Ekanem et al documented an SP rate of $1.4 \%$ in COVID-19 associated infection, with a strong male predominance, ${ }^{9}$ confirmed by Greenberg et al in a more recent study. ${ }^{10}$ These two studies included patients who were both non-ventilated and mechanically ventilated before being diagnosed with pneumothorax.

Regarding the risk of COVID-19 patients developing SP-SPM, Chong et al reported no connection with age, active smoking status, or pre-existing lung disease. ${ }^{8}$ Several reports showed a higher severity and worse outcome in patients with SP-SPM associated with SARS$\mathrm{CoV}-2 .^{6,8,11}$ It correlated with the severity of lung damage, rather than with the magnitude of SP-SPM. ${ }^{12-14}$ However, a multicenter retrospective case study by Martinelli et $\mathrm{al}^{7}$ challenged these findings, reporting an overall survival rate of $63.1 \%$ in patients confirmed with COVID-19 and $\mathrm{SP}^{7}$

We conducted a one-year review of the medical records in the emergency departments (ED) of two tertiary hospitals: Emergency Clinical Municipal Hospital and "Pius Brinzeu" Emergency Clinical County Hospital, both teaching hospitals affiliated with Victor Babes University of Medicine and Pharmacy in Timisoara. The aim of our retrospective research was twofold: (a) to investigate the characteristics of patients with SP-SPM (both with and without COVID-19) and compare them to patients with sole COVID-19; (b) to quantify the risk of in-hospital mortality associated with SP-SPM and COVID-19.

\section{Patients and Methods}

\section{Study Design and Patients}

The study design followed a retrospective case-control protocol: a review was carried out on 64,845 electronic medical records (EMRs) of patients admitted to the two EDs between 1st April 2020 and 31st March 2021. Seventy cases of SP-SPM were identified in the hospitals' computer systems through keywords-based automatic search. The results were then manually double-checked for compliance with the inclusion criteria and completeness of the essential required EMR data. They comprised the case group. For each case, two controls were included from consecutive patients admitted with SARS-CoV-2 infection at the same ED, immediately before (one) and after (one) the case patient. In the situation of two or more consecutive cases, the allocation was adjusted accordingly, keeping the same ratio of 2:1 between controls and cases. We assumed this approach would compensate for the effects of variability in pandemic waves' severity, ED crowding, shortage of medical personnel and other resources, and even the effects of medical professionals' burnout; it would also assure the comparability of the groups regarding other unknown or unforeseen factors.

Starting in March 2020, the hospital admittance protocols required all patients to undergo chest-imaging (either $\mathrm{X}$-ray or computed tomography) and a reverse transcription-polymerase chain reaction (RT-PCR) test for SARS$\mathrm{CoV}-2$ infection, irrespective of symptoms or diagnosis. A confirmed COVID-19 hospital encounter was defined under the diagnosis code U07.1 of the International Classification of Diseases, 10th Revision, Clinical Modification (ICD-10-CM) upon the patient's discharge from hospital. An encounter of SP-SPM was defined under the diagnosis code J93.11 (SP) or J98.2 (SPM) upon discharge.

The case group had the following inclusion criteria: aged 18 or older; presence of pneumothorax or pneumomediastinum on chest radiography or computed tomography (CT); RT-PCR test performed for SARS-CoV-2 infection. The exclusion criteria were as follows: aged below 18 years; post-traumatic pneumothorax or pneumomediastinum; invasive or non-invasive mechanical ventilation initiated before arrival at the ED; post-procedural pneumothorax or pneumomediastinum, such as thoracocentesis, or a central venous catheter in the jugular or subclavian veins.

Inclusion criteria for the control group were as follows: aged 18 or older; RT-PCR confirmation of SARS-CoV-2 infection in a nasopharyngeal swab. Age below 18 years was the only exclusion criterion for the control group. Within the control patients, some EMR data could not be retrieved for all the variables. There were two reasons for 
the missing values in the hospital information systems: (i) at the beginning of the pandemic, there was not a clear protocol for COVID-19 patients, so medical investigation was dependent on the doctor in charge; (ii) at certain times, the flow of patients in the ED overwhelmed the hospital's laboratory capacity or the availability of reactants for all the requested blood tests. Since the priority for data retrieval was to comply with the need to avoid any possible bias, the choice was made to keep the incomplete records in the working data set if the missing laboratory results were not essential to the study objectives. No data imputation was performed.

The primary outcome of our study was in-hospital mortality, a health indicator that captures the severity of the medical condition and the healthcare services' capacity of response. ${ }^{15,16}$ In-hospital death was defined as a medical encounter with discharge status of "died" or "died in a medical facility".

Based on the recently published data regarding $\mathrm{SP}^{6,17}$ and sample size criteria for binary logistic models, ${ }^{18}$ the necessary sample size was estimated to be 64 patients in the case group, for the following: alpha $=0.05$, beta $=0.2$, model based on conventional Maximum Likelihood (ML), $\mathrm{R}$-squared $=0.5$, and probability of the outcome in cases and controls equal to 0.3 and 0.1 , respectively. As the number of cases in the 12-month review of ED records exceeded this necessary size, the required statistical power was attained, so we proceeded with the data curation and formal analysis.

The study design is presented in Figure 1.

\section{Data Collection}

We collected data regarding: (a) demographics, comorbidities, symptoms, and vital signs on arrival at the ED; (b) laboratory tests and radiological findings on chest radiography and CT imaging. Other information of interest was related to the length of hospitalization, need for ICU admission, need for mechanical ventilation during the hospitalization, and treatment options for SP-SPM patients (eg, conservative-observation, chest drainage, or surgery). Collected data were de-identified before conducting the statistical analysis.

\section{Data Analysis}

Descriptive statistics included the observed frequency counts (percentage) for categorical variables and median (Inter Quartile Range) for numerical variables. Mean and standard deviation for numerical variables were also included (except for the laboratory results), regardless of distribution. Univariate non-parametric statistical tests

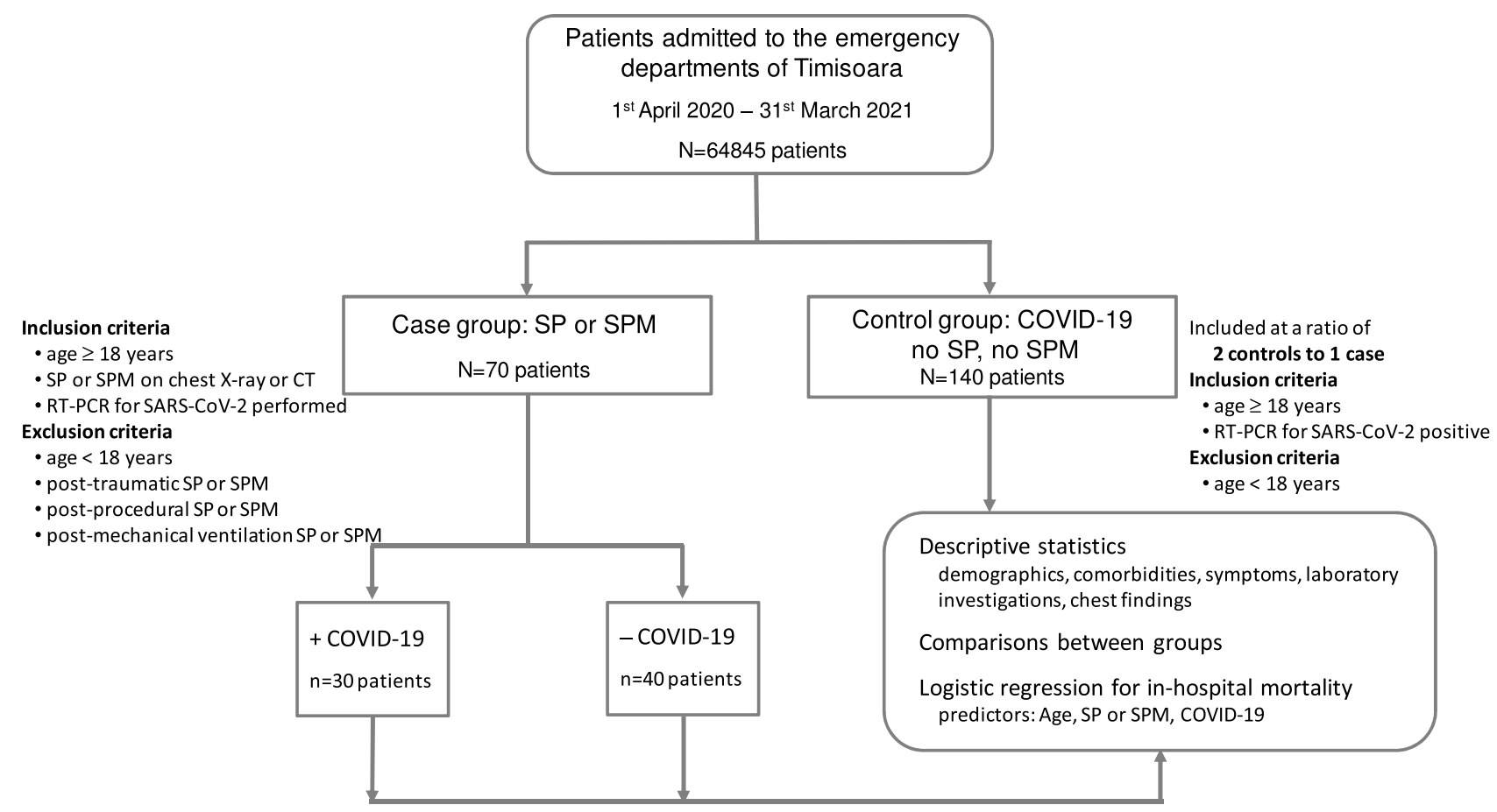

Figure I Study flow diagram.

Abbreviations: CT, computed tomography; RT-PCR, reverse transcription-polymerase chain reaction; SP, spontaneous pneumothorax; SPM, Spontaneous pneumomediastinum. 
were applied to compare the distribution of data across two or multiple groups, as appropriate (either MannWhitney U or Kruskal-Wallis tests, respectively). The Chi-square statistical test (either asymptotic or Monte Carlo simulation with 10,000 samples) was applied to check the statistical significance of the association between the categorical variables. The Shapiro-Wilk statistical test was employed to test for normality (all the numerical variables had non-normal distributions).

A step-wise logistic regression model was applied with mortality as the outcome, and age, a positive diagnosis of SPSPM or COVID-19 as possible independent predictors (controlling for gender and smoking status), based on the research hypothesis and the preliminary univariate analysis. The Akaike information criterion (AIC) was used to select the best fitting model when applying the logistic regression.

We conducted a post hoc sensitivity analysis, comprising: (a) a post hoc power analysis for the logistic regression model; (b) analysis of alternative regression models, employing different building strategies. ${ }^{19}$ For the power analysis, two approaches were considered: (a1) simple logistic regression based on observed proportions of the outcome in different groups; ${ }^{20,21}$ (a2) multiple logistic regression with binary and continuous predictors. ${ }^{22}$
The statistical analysis was conducted at a $95 \%$ level of confidence and 5\% level of statistical significance. All reported probability values were two-tailed.

The data analysis was performed using the statistical software IBM SPSS v. 25 and R v. 4.0.5 packages (including "webPower" v. 0.6 and "powerMediation" v. 0.3.4).

\section{Ethics}

The study was conducted in accordance with the Declaration of Helsinki, and the protocol was approved by the Ethics Committees of the Emergency Clinical Municipal Hospital in Timisoara (number E-1270/ 05.03.2021) and of the "Pius Brinzeu" Emergency Clinical County Hospital in Timisoara (number 229/ 26.02.2021). No informed consent was needed for this secondary use of medical data; therefore, the written patient consent was waived.

\section{Results}

\section{Characteristics of SP-SPM and COVID-19 Patients}

Within the 70 cases, there were 30 positive and 40 negative SARS-CoV-2 patients. Tables 1 and 2 present the

Table I Patients Enrolled: Descriptive Statistics for Demographics and Comorbidities

\begin{tabular}{|c|c|c|c|c|c|}
\hline Variable & $\begin{array}{l}\text { All Patients } \\
(\mathrm{N}=2 \mid 0)\end{array}$ & $\begin{array}{c}\text { SP or SPM } \\
\text { COVID-19 } \\
(N=40)\end{array}$ & $\begin{array}{l}\text { SP or SPM } \\
+ \text { COVID-19 } \\
(\mathrm{N}=30)\end{array}$ & $\begin{array}{c}\text { Neither SP nor SPM } \\
+ \text { COVID-19 } \\
(N=140)\end{array}$ & p-value ${ }^{(a),(b)}$ \\
\hline Age in years ${ }^{(a)}$ & $\begin{array}{l}57.91 \pm 15.21 \\
59.5(47-69)\end{array}$ & $\begin{array}{l}48.93 \pm 17.38 \\
49.5(34-62)\end{array}$ & $\begin{array}{l}64.43 \pm 12.24 \\
66.5(57-73)\end{array}$ & $\begin{array}{c}59.08 \pm 14.08 \\
60.5(48-69.5)\end{array}$ & $<0.001^{* *}$ \\
\hline Gender, male ${ }^{(b)}$ & $120(57.1 \%)$ & 27 (67.5\%) & 21 (70.0\%) & $72(51.4 \%)$ & 0.059 \\
\hline Active smoker ${ }^{(b)}$ & 81 (38.6\%) & $26(65.0 \%)$ & $6(20.0 \%)$ & 49 (35.0\%) & $<0.00 I^{* *}$ \\
\hline \multicolumn{6}{|l|}{ Comorbidities } \\
\hline COPD $^{(b)}$ & 14 (6.7\%) & 7 (I7.5\%) & 4 (I3.3\%) & $3(2.1 \%)$ & $0.002 * *$ \\
\hline Asthma (b) & $12(5.7 \%)$ & $2(5.0 \%)$ & $4(13.3 \%)$ & $6(4.3 \%)$ & 0.156 \\
\hline Hypertension (b) & 125 (59.5\%) & $10(25.0 \%)$ & $19(63.3 \%)$ & $96(68.6 \%)$ & $<0.001^{* *}$ \\
\hline Diabetes mellitus ${ }^{(b)}$ & $42(20.0 \%)$ & $3(7.5 \%)$ & $4(13.3 \%)$ & $35(25.0 \%)$ & $0.031 *$ \\
\hline Obesity ${ }^{(b)}$ & $36(17.1 \%)$ & I (2.5\%) & $2(6.7 \%)$ & $33(23.6 \%)$ & $0.002 * *$ \\
\hline CKD ${ }^{(b)}$ & 17 (8.I\%) & $2(5.0 \%)$ & $4(13.3 \%)$ & II (7.9\%) & 0.473 \\
\hline Active cancer ${ }^{(b)}$ & $10(4.8 \%)$ & 5 (I2.5\%) & $2(6.7 \%)$ & $3(2.1 \%)$ & $0.017^{*}$ \\
\hline
\end{tabular}

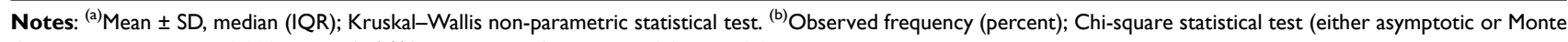
Carlo simulation with 10,000 samples). $* * *$ Statistical significance, $\mathrm{p}<0.05, \mathrm{p}<0.01$.

Abbreviations: CKD, chronic kidney disease; COPD, chronic obstructive pulmonary disease; IQR, inter-quartile range; SD, standard deviation; SP, spontaneous pneumothorax; SPM, spontaneous pneumomediastinum. 
Table 2 Patients Enrolled: Descriptive Statistics for Symptoms, Hospitalization Data, and Final Outcome

\begin{tabular}{|c|c|c|c|c|c|}
\hline Variable & $\begin{array}{l}\text { All Patients } \\
\qquad(\mathrm{N}=2 \mid 0)\end{array}$ & $\begin{array}{c}\text { SP or SPM } \\
\text { - COVID-19 } \\
(N=40)\end{array}$ & $\begin{array}{c}\text { SP or SPM } \\
+ \text { COVID-I9 } \\
(\mathrm{N}=30)\end{array}$ & $\begin{array}{c}\text { Neither SP nor SPM + } \\
\text { COVID-19 } \\
(\mathrm{N}=140)\end{array}$ & p-value (a),(b) \\
\hline Cough ${ }^{\text {(a) }}$ & 155 (73.8\%) & $16(40.0 \%)$ & $19(63.3 \%)$ & $120(85.7 \%)$ & $<0.00 I^{* *}$ \\
\hline Dyspnea (a) & 134 (63.8\%) & $35(87.5 \%)$ & $28(93.3 \%)$ & $7 \mathrm{l}(50.7 \%)$ & $<0.00 I^{* *}$ \\
\hline Chest pain (a) & $42(20.0 \%)$ & $24(60.0 \%)$ & $13(43.3 \%)$ & $5(3.6 \%)$ & $<0.001 * *$ \\
\hline Tiredness ${ }^{(a)}$ & $73(34.8 \%)$ & $12(30.0 \%)$ & $20(66.7 \%)$ & $4 \mathrm{I}(29.3 \%)$ & $<0.00 I^{* *}$ \\
\hline $\mathrm{SpO} 2$ on room air ${ }^{\text {(b) }}$ & $\begin{array}{l}89.18 \pm 8.72 \\
90(87-95)\end{array}$ & $\begin{array}{c}92.73 \pm 6.18 \\
95(89.5-97)\end{array}$ & $\begin{array}{c}77.67 \pm 14.56 \\
82(68-88)\end{array}$ & $\begin{array}{c}90.64 \pm 5.02 \\
90.5(88-95)\end{array}$ & $<0.00 I^{* *}$ \\
\hline Hospitalization in days ${ }^{(b)}$ & $\begin{array}{c}13.9 \pm 12 \\
12(8-16)\end{array}$ & $\begin{array}{l}10.68 \pm 7.46 \\
8(6-13.5)\end{array}$ & $\begin{array}{c}21.73 \pm 24.8 \mid \\
\mid 7.5(8-24)\end{array}$ & $\begin{array}{c}13.14 \pm 7.45 \\
12(9-15)\end{array}$ & $<0.001^{* *}$ \\
\hline $\begin{array}{l}\text { Days from the onset of } \\
\text { COVID-19 } \\
\text { symptoms to diagnosis of } \\
\text { SP-SPM } \\
\text { (b) }\end{array}$ & - & - & $\begin{array}{l}7.4 \pm 6.996 \\
4(2-10)\end{array}$ & - & - \\
\hline $\begin{array}{l}\text { Required mechanical } \\
\text { ventilation }^{(a)}\end{array}$ & 31 (22.14\%) & $3(7.5 \%)$ & II (36.7\%) & $17(24.3 \%)$ & $0.012 *$ \\
\hline Required ICU (a) & $35(16.7 \%)$ & $3(7.5 \%)$ & $14(46.7 \%)$ & $18(12.9 \%)$ & $<0.001^{* *}$ \\
\hline Deceased $^{(a)}$ & $44(21 \%)$ & $4(10 \%)$ & $14(46.7 \%)$ & $26(18.6 \%)$ & $<0.001^{* *}$ \\
\hline
\end{tabular}

Note: *** Statistical significance, $\mathrm{p}<0.05, \mathrm{p}<0.0 \mathrm{O}$. ${ }^{(a)}$ Observed frequency (percent); Chi-square statistical test (either asymptotic or Monte Carlo simulation with 10,000 samples). ${ }^{(b)}$ Mean $\pm \mathrm{SD}$, median (IQR); Kruskal-Wallis non-parametric statistical test.

Abbreviations: ICU, Intensive Care Unit; IQR, inter-quartile range; SD, standard deviation; SP, spontaneous pneumothorax; SPM, spontaneous pneumomediastinum; SpO2, peripheral oxygen saturation at hospital admittance.

descriptive statistics for demographics, comorbidities, symptoms, and final outcome. In SP-SPM patients, COVID-19 was frequently associated with male gender, older age, and non-smoking status.

The most common comorbidity in SP-SPM cases was hypertension; the most frequent symptom was dyspnea. The highest mortality and need for ICU admission were observed in SP-SPM cases with associated SARS-CoV-2 infection.

Laboratory findings are presented in Table 3. Hypoxia, hyperglycemia, elevated values for C-reactive protein, lactate dehydrogenase (LDH), and D-dimers were more common in SP-SPM patients with COVID-19.

Table 4 shows the imaging findings, extension degree of pneumothorax and ground glass opacity (GGO). Treatment options for the SP-SPM cases were included. Most SP-SPM patients were diagnosed by chest-CT, and the most frequent treatment for SP-SPM cases was thoracic drainage (either with or without COVID-19). None of the patients had compressive pneumothorax requiring needle decompression.
The location of pneumothorax was unilateral and predominantly right-sided (54.3\% of cases), regardless of COVID-19 status. Pneumomediastinum was found in a higher proportion of patients with associated SARS-CoV2 infection. Nine of the infected patients had only isolated pneumomediastinum without pneumothorax. Also, subcutaneous emphysema was significantly more frequently in COVID-19 cases compared to non-COVID-19 cases (43.3\% vs $7.5 \%$, respectively).

All patients with SPM were hospitalized for observation, with no need for further interventions: the SPM was minimal in all the cases (cervicotomy and mediastinal drainage were reserved for the patients with massive compressive SPM, or for those with massive subcutaneous emphysema). Surgical treatment was indicated in a higher percentage for non-COVID-19 patients (35\%) compared to the positive ones $(6.7 \%)$.

\section{Analysis of In-Hospital Mortality Risk}

Table 5 shows the results of the regression analysis with in-hospital mortality as the outcome. Models 0 , 1, and 2 
Table 3 Descriptive Statistics for Laboratory Investigations

\begin{tabular}{|c|c|c|c|c|c|}
\hline Variable & All Patients $(\mathrm{N}=2 \mid 0)$ & $\begin{array}{c}\text { SP or SPM } \\
\text { - COVID-19 } \\
(N=40)\end{array}$ & $\begin{array}{l}\text { SP or SPM } \\
+ \text { COVID-19 } \\
(\mathrm{N}=30)\end{array}$ & $\begin{array}{c}\text { Neither SP nor } \\
\text { SPM } \\
+ \text { COVID-19 } \\
(\mathrm{N}=140)\end{array}$ & $\begin{array}{l}\text { P-value } \\
\text { (a) }\end{array}$ \\
\hline C-reactive protein ${ }^{(a)}$ & $\begin{array}{c}n=\mid 81 \\
77.3(31.7-152)\end{array}$ & $\begin{array}{c}n=11 \\
34(8.65-81.75)\end{array}$ & $\begin{array}{c}n=30 \\
92.85(57-146)\end{array}$ & $\begin{array}{c}n=140 \\
70.25(30.9-156)\end{array}$ & $0.039 *$ \\
\hline Serum creatinine ${ }^{(a)}[\mathrm{mg} / \mathrm{dl}]$ & $0.84(0.7-1.04)$ & $0.845(0.74-1.065)$ & $1.005(0.8-1.34)$ & $0.8(0.695-1)$ & $0.046^{*}$ \\
\hline Urea $^{(\mathrm{a})}[\mathrm{mg} / \mathrm{dl}]$ & $37(28-58)$ & $31(25.5-42.5)$ & $45(32-66)$ & $37(27-60)$ & $0.044^{*}$ \\
\hline Leucocytes ${ }^{(a)}\left[\times 10^{3} / \mu \mathrm{L}\right]$ & $8.74(5.3-13.7)$ & II $(8.1-13.5)$ & $9.6(6.7-16.1)$ & $7.7(4.7-13.55)$ & $0.025^{*}$ \\
\hline Lymphocytes ${ }^{(\mathrm{a})}\left[\times 10^{3} / \mu \mathrm{L}\right]$ & $I(0.6-1.52)$ & $1.9(1.4-2.1)$ & $\mathrm{I}(0.7-\mathrm{I} .3)$ & $0.8(0.5-1.3)$ & $<0.001 * *$ \\
\hline Trombocytes ${ }^{(\mathrm{a})}\left[\times 10^{3} / \mu \mathrm{L}\right]$ & $248(179-340)$ & $302.5(242.5-360$ & $230(174-336)$ & $242(155.5-337)$ & $0.015^{*}$ \\
\hline D-dimer ${ }^{(a)}[\mathrm{ng} / \mathrm{mL}]$ & $\begin{array}{c}n=181 \\
780(460-1530)\end{array}$ & $\begin{array}{c}n=1 \mid \\
|49|(506-4496.5)\end{array}$ & $\begin{array}{c}n=30 \\
1681.5(932-2900)\end{array}$ & $\begin{array}{c}n=140 \\
655(435-1150)\end{array}$ & $<0.00 I^{* *}$ \\
\hline Serum glucose level ${ }^{(\mathrm{a})}[\mathrm{mg} / \mathrm{dl}]$ & $135.5(106-186)$ & $111(96.5-131.5)$ & $126(103-144)$ & $152(110.5-202.5)$ & $<0.001 * *$ \\
\hline $\operatorname{LDH}^{\left({ }^{(a)}\right.}[$ units/l] & $\begin{array}{c}n=164 \\
333.5(226.5-457.5)\end{array}$ & $\begin{array}{c}n=9 \\
188(180-208)\end{array}$ & $\begin{array}{c}n=15 \\
411(179-566)\end{array}$ & $\begin{array}{c}n=140 \\
337(236-447.5)\end{array}$ & $0.005^{* *}$ \\
\hline
\end{tabular}

Notes: Actual findings were recorded for every patient in the three groups, unless otherwise stated when values were missing. *** statistical significance, $\mathrm{p}<0.05, \mathrm{p}<0.0 \mathrm{I}$. (a)Median (IQR); either Kruskal-Wallis (for three independent groups) or Mann-Whitney $U$ (for two independent groups) non-parametric statistical tests were applied. Abbreviations: IQR, inter-quartile range; LDH, lactate dehydrogenase; SP, spontaneous pneumothorax; SPM, spontaneous pneumomediastinum.

were built applying a step-wise logistic regression and employing AIC for model selection. Model 2 explained about $33 \%$ of the mortality risk (Nagelkerke $\mathrm{R}$-square $=0.326$ ) and supported the significant risk contribution of age, presence of SP or SPM, and presence of COVID-19.

In all three models, the regression coefficient of age remained constant and highly significant: each additional year added a mortality risk of $9.4 \%$; the $95 \%$ CI for OR in Model 2 was (1.054-1.135).

Based on Model 2, the SP-SPM increases almost four times the risk of death, ie at a ratio of 3.758 while controlling for the other factors in the model. Similarly, for patients of the same age, gender, smoking habit, and SPSPM condition, the presence of COVID-19 increases the in-hospital mortality risk more than four times, ie at an OR of 4.412 .

\section{Sensitivity Analysis}

The study was powered based on data recently reported in the literature. The actual findings deviated from the initial assumptions, namely the observed proportion of the deceased in different groups, and the final model's R-squared. The post hoc power calculation considered two approaches, which led to different values: (1) based on the main predictor (ie SPSPM) in simple logistic regression, it was over 0.999; (2) based on the actual Nagelkerke R-squared, in multiple logistic regression with binary and continuous predictors, power resulted to be 0.894 .

We built alternative regression models by: (1) employing the Bayesian information criterion (instead of AIC) for model selection; (2) controlling for additional possible confounders. The results were similar to those of the primary analysis. In the supplementary Table S1, Model $\mathrm{S}$ is presented: compared to Model 2 in the primary analysis, it includes seven comorbidities as additional independent variables. Although Nagelkerke R-square slightly increased (as expected, due to the additional independent variables in the model), the AIC value was almost the same, as of Model 2. The regression coefficients and the resulting ORs for the age and SP-SPM were similar to those in Model 2. The risk associated with COVID-19 turned out to be higher, with an even larger 95\% CI. Model S would also suggest a significant risk associated with asthma, but not with the other comorbidities.

The fluctuation of the regression coefficients associated with COVID-19 points to a mediation or moderation role it may play in the outcome. However, such an 
Table 4 Chest Imaging Findings

\begin{tabular}{|c|c|c|c|c|c|}
\hline Variable & $\begin{array}{l}\text { All Patients } \\
(\mathrm{N}=2 \mid 0)\end{array}$ & $\begin{array}{c}\text { SP or SPM } \\
\text { - COVID-I9 } \\
(N=40)\end{array}$ & $\begin{array}{l}\text { SP or SPM } \\
+ \text { COVID-19 } \\
(N=30)\end{array}$ & $\begin{array}{c}\text { Neither SP nor SPM } \\
+ \text { COVID-19 } \\
(N=140)\end{array}$ & p-value (a) \\
\hline CT investigation ${ }^{(a)}$ & I 88 (89.5\%) & $25(62.5 \%)$ & $29(96.67 \%)$ & 134 (95.7\%) & - \\
\hline X-ray investigation (a) & 23 (11\%) & $16(40 \%)$ & I (3.3\%) & $6(4.3 \%)$ & - \\
\hline Interstitial lung infiltrates ${ }^{(a)}$ & 69 (32.9\%) & $6(15 \%)$ & $14(46.7 \%)$ & 49 (35\%) & $0.013^{*}$ \\
\hline Ground-glass opacities (a) & $156(74.3 \%)$ & 0 & 25 (83.3\%) & $|3|$ (93.6\%) & $<0.001 * *$ \\
\hline Right pneumothorax ${ }^{(a), \#}$ & 38 & $22(55 \%)$ & $16(53.3 \%)$ & - & (b) 0.890 \\
\hline Left pneumothorax ${ }^{(a), \#}$ & 25 & $18(45 \%)$ & 7 (23.3\%) & - & (b) 0.061 \\
\hline Pneumomediastinum ${ }^{(a)}$ & 20 & $4(10.0 \%)$ & $16(53.3 \%)$ & - & (b) $<0.00 I^{* *}$ \\
\hline Subcutaneous emphysema ${ }^{(a)}$ & 16 & $3(7.5 \%)$ & $13(43.3 \%)$ & - & (b) $<0.00 I^{* *}$ \\
\hline \multicolumn{6}{|l|}{ Extension of pneumothorax ${ }^{\text {(a) }}$} \\
\hline No pneumothorax & - & I (2.5\%) & $9(30 \%)$ & - & \multirow{4}{*}{ (b) $0.006 * *$} \\
\hline$<10 \%$ & - & $8(20 \%)$ & 7 (23.3\%) & - & \\
\hline $10-50 \%$ & - & 15 (37.5\%) & $5(16.7 \%)$ & - & \\
\hline$>50 \%$ & - & $16(40 \%)$ & $9(30 \%)$ & - & \\
\hline \multicolumn{6}{|c|}{ Extension of ground glass opacity ${ }^{\text {(a) }}$} \\
\hline No ground glass opacity & 6 & - & $4(13.3 \%)$ & $2(1.5 \%)$ & \multirow{4}{*}{ (c) $0.002 * *$} \\
\hline$<20 \%$ & 48 & - & $4(13.3 \%)$ & 44 (33.6\%) & \\
\hline $20-50 \%$ & 54 & - & 7 (23.3\%) & 47 (35.9\%) & \\
\hline$>50 \%$ & 53 & - & 15 (50\%) & 38 (29\%) & \\
\hline \multicolumn{6}{|l|}{ Treatment ${ }^{\text {(a) }}$} \\
\hline Observation & 14 & I (2.5\%) & $13(43.3 \%)$ & - & (b) $<0.00 I^{* *}$ \\
\hline Chest tube & 50 & 33 (82.5\%) & 17 (56.7\%) & - & (b) $0.018^{*}$ \\
\hline Surgery & 16 & 14 (35\%) & $2(6.7 \%)$ & - & (b) $0.005^{* *}$ \\
\hline
\end{tabular}

Notes: Treatment options were recorded for patients with spontaneous pneumothorax (SP) or spontaneous pneumomediastinum (SPM). ${ }^{(a)}$ Observed frequency (percent); Chi-square statistical test (either asymptotic or Monte Carlo simulation with 10,000 samples). ${ }^{(b)}$ Chi-square statistical test for the two sub-groups of SP-SPM cases only. (c) Chi-square statistical test for the sub-groups $\left\{\right.$ SP or SPM + COVID-19\} and \{neither SP nor SPM + COVID-19\}. ${ }^{\#}$ One $\{$ SP or SPM - COVID-19\} case had both right and left SP, and no SPM; two \{SP or SPM + COVID-19\} cases had both right and left pneumothorax, and SPM. *,* statistical significance, $p<0.05, p<0.01$.

Abbreviations: CT, computed tomography; SP, spontaneous pneumothorax; SPM, spontaneous pneumomediastinum; X-ray, X-rays chest radiography.

investigation is beyond the present analysis and would require a larger study with a different design. Model S would open possible directions for further investigation, with the caveat concerning the ratio between the model's independent predictors and the number of dataset records (ie, patients observed), as for any multiple regression analysis. $^{23}$
When building a logistic regression model, a parsimonious alternative is preferred, and possible predictors are purposefully selected grounded on research hypotheses, preliminary descriptive statistics, and medical meaningfulness. Based on AIC and the analysis of alternative regression models, Model 2 remained robust and reliable. 
Table 5 The Logistic Regression Model for In-Hospital Mortality

\begin{tabular}{|c|c|c|c|}
\hline \multicolumn{4}{|c|}{ Model 0: Deceased Age + GenderM + ActiveSmoker } \\
\hline Predictor & B \pm Std. Err & p-value & $\operatorname{Exp}(B)(95 \% \mathrm{Cl})$ \\
\hline Age & $0.091 \pm 0.018$ & $<0.001 * *$ & $1.096(1.057-1.136)$ \\
\hline GenderM & $0.206 \pm 0.39$ & 0.597 & NA \\
\hline ActiveSmoker & $0.057 \pm 0.456$ & 0.901 & NA \\
\hline & \multicolumn{3}{|c|}{$\mathrm{AIC}_{0}=182.23,4 \mathrm{df} ;$ Nagelkerke R-square $=0.279$} \\
\hline \multicolumn{4}{|c|}{ Model I: Deceased $\sim$ Age + SP or SPM Controlling for: GenderM + ActiveSmoker } \\
\hline Predictor & B \pm Std. Err & p-value & $\operatorname{Exp}(B)(95 \% \mathrm{Cl})$ \\
\hline Age & $0.091 \pm 0.018$ & $<0.00 I^{* *}$ & $1.096(1.057-1.136)$ \\
\hline SP or SPM & $0.761 \pm 0.412$ & $0.064^{\#}$ & $2.14(0.955-4.795)$ \\
\hline & \multicolumn{3}{|c|}{$\left.\mathrm{AIC}_{1}=180.8 \mathrm{I}, 5 \mathrm{df} ;\left(\mathrm{AIC}_{1}, \mathrm{AIC}\right)_{0}\right), \mathrm{p}=0.064^{\#} ;$ Nagelkerke R-square $=0.299$} \\
\hline \multicolumn{4}{|c|}{ Model 2: Deceased Age + SP or SPM + COVID-19 Controlling for: GenderM + ActiveSmoker } \\
\hline Predictor & B \pm Std. Err & p-value & $\operatorname{Exp}(\mathrm{B})(95 \% \mathrm{Cl})$ \\
\hline Age & $0.090 \pm 0.019$ & $<0.00 I^{* *}$ & $1.094(1.054-1.135)$ \\
\hline SP or SPM & $1.324 \pm 0.489$ & $0.007^{* *}$ & $3.758(1.443-9.792)$ \\
\hline COVID-19 & $1.484 \pm 0.728$ & $0.041^{*}$ & $4.412(1.060-18.370)$ \\
\hline
\end{tabular}

Notes: A step-wise analysis was conducted, based on the Akaike information criterion: only variables that remained in the model are shown. In Model I, the presence of SP or SPM was marginally significant. In Model 2, which included COVID-19, the presence of SP or SPM is highly significant. Exp (B) is equivalent to the odds ratio (OR), a measure of a relationship's strength between the predictor and the binary outcome. ${ }^{\#}$ Marginal statistical significance, $\mathrm{P}<0.1 ; *$ statistical significance, $\mathrm{P}<0.05 ; * *$ high statistical significance, $\mathrm{p}<0.01$.

Abbreviations: AIC, Akaike information criterion; B \pm Std. err, coefficient of regression \pm standard error; $\mathrm{Cl}$, confidence interval; df, degrees of freedom; Gender $\mathrm{M}$, gender male; NA, not applicable; SP, spontaneous pneumothorax; SPM, spontaneous pneumomediastinum.

\section{Discussion}

Underlying pulmonary diseases may frequently cause SP and SPM. In the literature, pathologies, such as COPD, tuberculosis, lung cancer, cystic fibrosis and Pneumocystis jirovecii pneumonia are reported as responsible for development of SP-SPM. ${ }^{1,24}$ In our study, only small percentages of patients were admitted to the EDs with these conditions: $13.3 \%$ COPD, $13.3 \%$ asthma, and $6.7 \%$ lung cancer. This might suggest that SARS-CoV-2 infection alone was a major predisposing factor for SP-SPM development, but studies specifically designed to test this hypothesis are needed.

We also noticed a higher proportion of male SP-SPM cases, both in COVID-19 (70\%) and in non-COVID-19 patients $(67.5 \%)$, which was consistent with the medical literature data. In pre-pandemic studies, the SP incidence was higher in males ( 7.4 to 18 cases per 100,000 population) compared to females (1.2 to 6 cases per 100,000 population). ${ }^{1}$ A higher SP proportion in males was also highlighted in studies on patients with SARS-CoV-2 infection. $6,7,9,11$

Cigarette smoking is another acknowledged risk factor for developing SP-SPM. ${ }^{1,24}$ In our study, $65 \%$ of the SPSPM cases with no SARS-CoV-2 infection were active smokers. Among the cases confirmed with COVID-19, only $20 \%$ were active smokers, suggesting that smoking was a non-contributory individual risk factor for SP-SPM complications in patients with SARS-CoV-2 infection.

High blood pressure (HBP) was by far the most common comorbidity found in all patients. Comparing HBP frequency in the three groups, it seemed to be associated with SARS-CoV-2 infection, rather than SP-SPM condition. Further investigations are needed to confirm this hypothesis, since in our present study, the age of patients with SP-SPM and COVID-19 was significantly higher compared to cases without COVID-19 (median values of 66.5 and 49.5, respectively) and HBP risk increases with age. $^{25}$ 
Cough, a common symptom of COVID-19, might trigger SP-SPM, ${ }^{1,24}$ but it was more frequent in the COVID19 control group, compared to SARS-CoV-2 positive cases (85.7\% and $63.3 \%$, respectively).

In an observational cohort study by Qu et al, ${ }^{26}$ dyspnea was found as a marker for critical forms of COVID-19. An association among chest distress, dyspnea, and shortness of breath was related to increased mortality. ${ }^{26}$ In our research, dyspnea was the most common symptom associated with SP-SPM (93.3\%), but it was also present in a high percentage of the COVID-19 control group (50.7\%). Chest pain was found to be the most specific symptom in the SP-SPM case group, regardless of COVID-19 condition: present in $60 \%$ of negative vs $43.3 \%$ of positive cases. In the control group (COVID-19 positive, non-SPSPM), chest pain occurred significantly less (3.6\%). Therefore, chest pain in patients with SARS-CoV-2 infection would indicate a possible SP-SPM complication.

The peripheral oxygen saturation in room air at hospital admittance (SpO2) corroborated our findings on prolonged hospitalization, higher percentage of ICU admission, and higher mortality: $\mathrm{SpO} 2$ had the lowest values among the cases associated with COVID-19 (SPSPM with COVID-19), who also had the most dramatic rate of death (almost 47\%). Low $\mathrm{SpO} 2$ is acknowledged as a consequence of SARS-CoV-2 induced pulmonary infiltration. Tabernero et $\mathrm{al}^{27}$ reported a multivariate Cox regression model developed based on data of 513 patients hospitalized for COVID-19 that identified six independent factors associated with poor outcome, among which the low oxygen saturation was the most significant. In a retrospective observational study, Liu et $\mathrm{al}^{28}$ evaluated the risk prediction tools for patients with COVID-19 in Wuhan, China, and they showed that respiration rate score, oxygen saturations score, temperature score, and AVPU (Alert, Voice, Pain, Unresponsive) score were independent predictors of death. Still, after adjusting for other variables, low oxygen saturation and heart rate did not predict death. Although the underlying mechanisms and effective solutions need further investigations, $\mathrm{SpO} 2$ remains an important factor in the patients' triage. In addition to the vital parameters used for risk stratification in COVID-19 patients, radiological predictors have been used in some studies to quantify the risk for developing complications such as SP-SPM in hospitalized patients with SARS-CoV2 infection. Palumbo et $\mathrm{al}^{29}$ tracked the Macklin effect on chest CT in COVID-19 patients and reported it as an early detector of lung frailty.
The mean time from the onset of COVID-19 symptoms until the ED diagnosis of SP-SPM was 7.4 days, with high variability indicated by a standard deviation of the same magnitude ( $\mathrm{SD}=6.996$ days). On the one hand, this delay in admission to hospital could be explained by the late onset of SP-SPM; on the other hand, fear and distrust of the health system, developed by patients during the pandemic, might generate such late admissions. The high variability would support the latter hypothesis, at least for part of the cases.

The chest CT was the most common imaging method used for SP-SPM diagnosis in the ED. Among the SPSPM cases associated with SARS-CoV-2 infection, only $30 \%$ had extended pneumothorax (ie over $50 \%$ of the lung fields), while $50 \%$ had extended ground-glass lesions. Increased mortality in this group could have been associated with the spread of ground-glass lesions, rather than the size of pneumothorax. This finding was consistent with other published data. ${ }^{6-8}$ The extended ground-glass lesions were significantly more frequent in the SP-SPM cases associated with SARS-CoV-2 infection, than the COVID19 control patients. Moreover, the inflammatory syndrome associated with COVID-19-specific GGO led to adhesions between the lung and parietal pleura, which would explain the higher frequency of partial pneumothorax. ${ }^{30}$ A causal relationship between these lesions and the SP-SPM development cannot be inferred, but the frequent association between GGO and SP-SPM was noticeable. On the other hand, $13.3 \%$ of SP-SPM cases with COVID-19 did not have GGO on chest imaging. This further suggested that possible additional risk factors, apart from COVID-19 inflammatory syndrome, may have been involved in the SP-SPM mechanism. The alveolar wall rupture due to the virus-induced frailty of airways' tissue and increased pressure caused by the cough effort might be one of the causes. ${ }^{29}$ Also, microvascular thrombosis and the cytokine storm were associated with more severe forms of the disease and considered main contributors of such complications. $^{29,31}$

Other important chest $\mathrm{CT}$ or X-ray findings were pulmonary interstitial infiltrates, identified mainly in SP-SPM cases with COVID-19, compared to non-COVID-19 cases ( $46.7 \%$ vs $15 \%$, respectively). Pathological findings, such as early fibrosis, cyst formation, traction and air-leak described in the evolution of COVID-19 patients, were reported as related to the SP development. ${ }^{32}$ Our study included only patients who were neither mechanically ventilated nor exposed to iatrogenic procedures before 
their ED presentation, thus reliably excluding such causes for the observed SP-SPM cases.

Hospitalization was significantly longer for SP-SPM cases with COVID-19, and the percentage of ICU admission was significantly higher for them. Even more dramatically, the proportion of deceased people in the same group was more than double that of the COVID-19 controls. By themselves, these findings suggested a greater risk of in-hospital mortality in patients with SP-SPM associated with COVID-19. The logistic regression found a risk of death almost four times higher for SP-SPM cases compared to individuals of the same age, gender, smoking status, and SARS-CoV-2 infection. COVID-19 pneumonia brought a significant mortality risk of more than four times higher (although the 95\% confidence interval was quite large, due to the limited sample size of the study). In the regression model of mortality, age was also a significant predictor, each additional year contributing with $9.4 \%$ increase in the risk of in-hospital death. Our results were consistent with the current medical literature, in which SP related to COVID-19 pneumonia is associated with prolonged hospitalization, increased admission in ICU, and higher level of mortality. $6,8,33,34$

COVID-19 ARDS patients could be more vulnerable to SP-SPM complications compared with other causes of ARDS. ${ }^{29}$ The presence of pneumothorax in patients with Middle East respiratory coronavirus syndrome (MERS) or H1N1 was shown to be a negative prognostic factor. ${ }^{31,35}$

Taking all of the above into consideration, the association of SP-SPM with COVID-19 might still be underreported. Its prevalence, risk factors, and final outcome remain unclear. ${ }^{13,14,30-34,36-39}$ Our research contributes to understanding the combination of SP, SPM and COVID-19 in non-ventilated patients, and quantifying their associated risks for in-hospital mortality.

\section{Limitations}

Our study had some limitations, mainly generated by its retrospective design as an ED medical records review. First, since the group of cases was evaluated in the pandemic year, there might have been patients false negative for SARS-CoV-2, because testing was conducted too early or too late in the disease evolution. Second, tests for important inflammatory markers in COVID-19 (such as interleukin-6, ferritin, or procalcitonin) are not routinely conducted in EDs, therefore a correlation between SPSPM and COVID-19 inflammatory syndrome could not be investigated in this retrospective research.
Despite these limitations, the post hoc sensitivity analysis supports the robustness of our results. The consistency between the primary logistic regression and the sensitivity analysis confirmed our findings' credibility.

\section{Conclusion}

SP-SPM is a serious complication of SARS-CoV-2 infection that increases the risk of in-hospital mortality by almost four times. Its association with COVID-19 pneumonia led to prolonged hospitalization and a high risk of fatal outcomes, especially among the elderly. Chest pain and dyspnea in patients with SARS-CoV-2 infection requires an urgent investigation to rule out SP-SPM.

\section{Abbreviations}

ARDS, acute respiratory distress syndrome; COPD, chronic obstructive pulmonary disease; COVID-19, coronavirus disease 2019; CT, computed tomography; ED, emergency department; EMR, electronic medical record; GGO, ground glass opacity; HBP, high blood pressure; NA, not available or not applicable; OR, odds ratio; RTPCR, reverse transcription-polymerase chain reaction; SARS-CoV-2, severe acute respiratory syndrome-coronavirus 2; SD, standard deviation; $\mathrm{SP}$, spontaneous pneumothorax; SPM, spontaneous pneumomediastinum; SPSPM, SP or SPM; SpO2, peripheral oxygen saturation on room air, at hospital admittance.

\section{Institutional Review Board Statement}

The study was conducted according to the guidelines of the Declaration of Helsinki, and approved by the Ethics Committee of the Emergency Clinical Municipal Hospital Timisoara, code E-1270/05.03.2021 and of Emergency Clinical County Hospital "Pius Brinzeu" Timisoara, code 229/26.02.21.

\section{Data Sharing Statement}

The data sets are not publicly available, but de-identified data may be provided upon request from Dr. Adina Maria Marza.

\section{Informed Consent Statement}

Patient consent was waived due to the fact that the research implied the secondary use of medical data and statistical analysis was performed on de-identified data. 


\section{Acknowledgments}

The authors kindly acknowledge the colleagues who volunteered to participate in data collection.

\section{Author Contributions}

All authors made a significant contribution to the work reported, whether that is in the conception, study design, execution, acquisition of data, analysis and interpretation, or in all these areas; took part in drafting, revising or critically reviewing the article; gave final approval of the version to be published; have agreed on the journal to which the article has been submitted; and agree to be accountable for all aspects of the work.

\section{Funding}

This research received no external funding.

\section{Disclosure}

The authors report no conflicts of interest in this work.

\section{References}

1. Noppen M. Spontaneous pneumothorax: epidemiology, pathophysiology and cause. Eur Respir Rev. 2010;19(117):217-219. doi:10.1183/ 09059180.00005310

2. Zhu H, Wei L, Niu P. The novel coronavirus outbreak in Wuhan, China. Glob Heal Res Policy. 2020;5(1). doi:10.1186/s41256-020-00135-6

3. Hu B, Guo H, Zhou P, Shi ZL. Characteristics of SARS-CoV-2 and COVID-19. Nat Rev Microbiol. 2021;19(3):141-154. doi:10.1038/ s41579-020-00459-7

4. Palumbo D, Campochiaro C, Belletti A, et al. Pneumothorax/ Pneumomediastinum in non-intubated COVID-19 patients: differences between first and second Italian pandemic wave. Eur J Intern Med. 2021;88:144-146. doi:10.1016/j.ejim.2021.03.018

5. Guven BB, Erturk T, Kompe Ö, Ersoy A. Serious complications in COVID-19 ARDS cases: pneumothorax, pneumomediastinum, subcutaneous emphysema and haemothorax. Epidemiol Infect. 2021;149: e137. doi:10.1017/S0950268821001291

6. Miró Ò, Llorens P, Jiménez S, et al. Frequency, risk factors, clinical characteristics, and outcomes of spontaneous pneumothorax in patients with coronavirus disease 2019: a case-control, emergency medicine-based multicenter study. Chest. 2021;159(3):1241-1255. doi:10.1016/j.chest.2020.11.013

7. Martinelli AW, Ingle T, Newman J, et al. COVID-19 and pneumothorax: a multicentre retrospective case series. Eur Respir J. 2020;56(5):2002697. doi:10.1183/13993003.02697-2020

8. Chong WH, Saha BK, Hu K, Chopra A. The incidence, clinical characteristics, and outcomes of pneumothorax in hospitalized COVID-19 patients: a systematic review. Hear Lung. 2021;50 (5):599-608. doi:10.1016/j.hrtlng.2021.04.005

9. Ekanem E, Podder S, Donthi N, et al. Spontaneous pneumothorax: an emerging complication of COVID-19 pneumonia. Hear Lung. 2021;50(3):437-440. doi:10.1016/j.hrtlng.2021.01.020

10. Greenberg DJ, Nabors C, Chandy D, Dhand A. Pneumothorax and pneumomediastinum in patients hospitalized with coronavirus disease 2019 (COVID-19). Heart Lung. 2021;50(3):386-387. doi:10.1016/j. hrtlng.2021.02.006
11. Cabrera Gaytán DA, Pérez Andrade Y, Espíritu Valenzo Y. Pneumothorax due to COVID-19: analysis of case reports. Respir Med Case Rep. 2021;34:101490. doi:10.1016/j.rmcr.2021.101490

12. López Vega JM, Parra Gordo ML, Diez Tascón A, Ossaba Vélez S. Pneumomediastinum and spontaneous pneumothorax as an extrapulmonary complication of COVID-19 disease. Emerg Radiol. 2020;27 (6):727-730. doi:10.1007/s10140-020-01806-0

13. Marza AM, Petrica A, Buleu FN, Mederle OA. Case report: massive spontaneous pneumothorax - a rare form of presentation for severe COVID-19 pneumonia. Medicina. 2021;57(2):82. doi:10.3390/ medicina57020082

14. Shan S, Guangming L, Wei L, Xuedong Y. Spontaneous pneumomediastinum, pneumothorax and subcutaneous emphysema in COVID19: case report and literature review. Rev Inst Med Trop Sao Paulo. 2020;62:e76. doi:10.1590/S1678-9946202062076

15. Gray WK, Navaratnam AV, Day J, et al. Variability in COVID-19 inhospital mortality rates between national health service trusts and regions in England: a national observational study for the getting it right first time programme. EClinicalMedicine. 2021;35:100859. doi:10.1016/j.eclinm.2021.100859

16. Aragón MJ, Chalkley M. How do time trends in inhospital mortality compare? A retrospective study of England and Scotland over 17 years using administrative data. BMJ Open. 2018;8(2):e17195. doi:10.1136/bmjopen-2017-017195

17. Miró Ò, Jiménez S, Mebazaa A, et al. Pulmonary embolism in patients with COVID-19: incidence, risk factors, clinical characteristics, and outcome. Eur Heart J. 2021;42(33):3127-3142. doi:10.1093/eurheartj/ehab314

18. van Smeden M, Moons KGM, de Groot JAH, et al. Sample size for binary logistic prediction models: beyond events per variable criteria. Stat Methods Med Res. 2019;28(8):2455-2474. doi:10.1177/ 0962280218784726

19. Thabane L, Mbuagbaw L, Zhang S, et al. A tutorial on sensitivity analyses in clinical trials: the what, why, when and how. BMC Med Res Methodol. 2013;13(1):92. doi:10.1186/1471-2288-13-92

20. Demidenko E. Sample size determination for logistic regression revisited. Stat Med. 2007;26(18):3385-3397. doi:10.1002/sim.2771

21. Zhang Z, Yuan KH. Practical Statistical Power Analysis Using WebPower and R. Notre Dame. Indiana, USA: ISDSA Press; 2018.

22. Hsieh FY, Bloch DA, Larsen MD. A simple method of sample size calculation for linear and logistic regression. Stat Med. 1998;17 (14):1623-1634. doi:10.1002/(SICI)1097-0258(19980730)17:14<162 3::AID-SIM871>3.0.CO;2-S

23. Altman DG. Practical Statistics for Medical Research. London: Chapman and Hall; 1991.

24. Dionísio P, Martins L, Moreira S, et al. Spontaneous pneumomediastinum: experience in 18 patients during the last 12 years. $J$ Bras Pneumol. 2017;43(2):101-105. doi:10.1590/s1806-37562016000 000052

25. Buford TW. Hypertension and aging. Ageing Res Rev. 2016;26:96111. doi:10.1016/j.arr.2016.01.007

26. Qu G, Chen J, Huang G, et al. A quantitative exploration of symptoms in COVID-19 patients: an observational cohort study. Int $J$ Med Sci. 2021;18(4):1082-1095. doi:10.7150/ijms.53596

27. Tabernero E, Ruiz LA, España PP, et al. COVID-19 in young and middle-aged adults: predictors of poor outcome and clinical differences. Infection. 2020. PMID: 34463951. doi:10.1007/s15010-02101684-9

28. Liu FY, Sun XL, Zhang Y, et al. Evaluation of the risk prediction tools for patients with coronavirus disease 2019 in Wuhan, China: a single-centered, retrospective, observational study. Crit Care Med. 2020;48(11):e1004-e1011. doi:10.1097/CCM.0000000000004549

29. Palumbo D, Zangrillo A, Belletti A, et al.; COVID-BioB Study Group. A radiological predictor for pneumomediastinum/pneumothorax in COVID-19 ARDS patients. J Crit Care. 2021;66:1419. doi:10.1016/j.jcrc.2021.07.022. 
30. Blanc K, Bonnet N, Ouedraogo E, Arnaout M, Patout M, TandjaouiLambiotte Y. Interstitial lung disease-related pneumomediastinum in COVID-19 patients. ERJ Open Res. 2021;7(1):00014-2021. doi:10.1183/23120541.00014-2021

31. Ulutas H, Celik MR, Gulcek I, et al. Management of spontaneous pneumothorax in patients with COVID-19. Interact Cardiovasc Thorac Surg. 2021. doi:10.1093/icvts/ivab280

32. Owens C, Amin H, Alwan S, Mitra I. Delayed spontaneous pneumothorax presentation in Covid-19: case series and review. Front Int J Gen Med. 2021;14:2989-3000. doi:10.47746/FMCR.2020.1603

33. Kawachi J, Koyama H, Aida Y. Pneumothorax with coronavirus disease 2019 in non-ventilated patients: a single-center retrospective case series. Authorea Preprints. 2021. PPR: PPR368231. doi:10.22541/au.162602191.11443821/v1.

34. Chopra A, Al-Tarbsheh AH, Shah NJ, et al. Pneumothorax in critically ill patients with COVID-19 infection: incidence, clinical characteristics and outcomes in a case control multicenter study. Respir Med. 2021;184:106464. doi:10.1016/j.rmed.2021.106464

35. Bor C, Demirağ K, Uyar M, Cankayalı I, Moral AR. Recurrent spontaneous pneumothorax during the recovery phase of ARDS due to H1N1 infection. Balkan Med J. 2013;30(1):123-125. doi:10.5152/ balkanmedj.2012.086
36. Al-Shokri SD, Ahmed AOE, Saleh AO, AbouKamar M, Ahmed K, Mohamed MFH. Case Report: COVID-19-related pneumothoraxcase series highlighting a significant complication. Am J Trop Med Hyg. 2020;103(3):1166-1169. doi:10.4269/ajtmh.20-0713

37. Cut TG, Tudoran C, Lazureanu VE, Marinescu AR, Dumache R, Tudoran M. Spontaneous pneumomediastinum, pneumothorax, pneumopericardium and subcutaneous emphysema-Not so uncommon complications in Patients with COVID-19 pulmonary infection-A series of cases. J Clin Med. 2021;10(7):1346. doi:10.3390/jcm10071346

38. Shaikh N, Al Ameri G, Shaheen M, et al. Spontaneous pneumomediastinum and pneumothorax in COVID-19 patients: a tertiary care experience. Health Sci Rep. 2021;4(3):e339. doi:10.1002/hsr2.339

39. Shaikh N, Al Ameri G, Shaheen M, et al. Tension Pneumomediastinum: a life-threatening condition in patients with COVID-19. Qatar Med J. 2021;2021(3):55. doi:10.5339/ qmj.2021.55
International Journal of General Medicine

\section{Publish your work in this journal}

The International Journal of General Medicine is an international, peer-reviewed open-access journal that focuses on general and internal medicine, pathogenesis, epidemiology, diagnosis, monitoring and treatment protocols. The journal is characterized by the rapid reporting of reviews, original research and clinical studies

\section{Dovepress}

across all disease areas. The manuscript management system is completely online and includes a very quick and fair peer-review system, which is all easy to use. Visit http://www.dovepress.com/ testimonials.php to read real quotes from published authors. 